\title{
STUDY OF SPINAL ANAESTHESIA IN PERCUTANEOUS NEPHROLITHOTOMY (PCNL)
}

\author{
Abeezar Hussain ${ }^{1}$, Deepak Sethi² \\ ${ }^{1}$ Assistant Professor, Department of Anaesthesiology, American International Institute of Medical Sciences, Udaipur, Rajasthan. \\ ${ }^{2}$ Consultant Surgeon, Department of Surgery, RNT Medical College, Udaipur, Rajasthan.
}

\section{ABSTRACT}

\section{BACKGROUND}

Anaesthesia during Percutaneous Nephrolithotomy (PCNL) for staghorn stones is a challenge because of the possibility of fluid absorption, dilutional anaemia, hypothermia, or significant blood loss.[2] PCNL can be done under General Anaesthesia or Spinal Anaesthesia. The most important advantages of spinal anaesthesia are the decrease in intra-operative blood loss and consequently improving operating conditions, the decrease in peri-operative cardiac ischaemic incidents, post-operative hypoxic episodes, arterial and venous thrombosis and to provide proper post-operative pain control.

\section{MATERIALS AND METHODS}

50 Patients, older than 15 years of age, who were undergoing Percutaneous Nephrolithotomy (PCNL) in American International Institute of Medical Sciences, Udaipur were selected for study. Intra-operative and post-operative haemodynamic study and anaesthetic complications, amount of blood loss and surgeon/patient satisfaction were studied.

\section{RESULTS}

The mean age of patient was $40 \pm 15$ years; the mean calculus size was $50.5 \pm 9.5 \mathrm{~mm}$. The mean operative time is $90 \pm 30$ minutes. Return of sensory and motor activity took $140 \pm 50$ minutes. During the first part of anaesthesia, 10 patients developed bradycardia, 5 patients developed hypotension which were treated. Six patients complained of mild-to-moderate headache, dizziness, mild low back pain for 2 to 4 days after operation, which improved with analgesic and bed rest. The mean haemoglobin decrease during 24 postoperative hours was $2 \pm 4 \mathrm{~g} / \mathrm{dL}$, only 3 patients required transfusion of 1 unit of packed cell. The incidence of post-operative nausea/vomiting was observed in 5 patients. Surgeon's and Patient's satisfaction level was good after regional anaesthesia.

\section{CONCLUSION}

Spinal anaesthesia could be a very good method of anaesthesia for percutaneous nephrolithotomy with trivial pain, less blood loss and without major complications; and also surgeon's and patient's satisfaction is more. Thus, it may be good alternative of General Anaesthesia for performing PCNL in adult patients. Further studies may be carried out to statistically prove that Spinal Anaesthesia may be a better alternative to General Anaesthesia.

\section{KEYWORDS}

Spinal Anaesthesia, Percutaneous Nephrolithotomy, PCNL, Renal Calculus.

HOW TO CITE THIS ARTICLE: Hussain A, Sethi D. Study of spinal anaesthesia in percutaneous nephrolithotomy (PCNL). J. Evolution Med. Dent. Sci. 2016;5(92):6816-6819, DOI: 10. 14260/jemds/2016/1542

\section{BACKGROUND}

Percutaneous Nephrolithotomy (PCNL) is now popular method for removal of kidney and upper ureteric calculi. Percutaneous nephrolithotomy (PCNL) is the preferred treatment modality for renal calculi with a large stone burden (example, staghorn calculi) and renal calculi which have failed extracorporeal shockwave lithotripsy (ESWL).[1]

Anaesthesia during PCNL for staghorn stones is a challenge because of the possibility of fluid absorption, dilutional anaemia, hypothermia, or significant blood loss.[2]

An acceptable anaesthetic technique must have characteristics such as rapid onset and reversal of effects.

Financial or Other, Competing Interest: None.

Submission 23-09-2016, Peer Review 06-11-2016,

Acceptance 12-11-2016, Published 17-11-2016.

Corresponding Author:

Dr. Deepak Sethi,

\# 303, Akshansh Apartment,

Keshav Nagar-313001,

Udaipur.

E-mail: deepanjali.d1972@gmail.com

DOI: $10.14260 /$ jemds/2016/1542

\section{(c) $(\mathrm{i})($}

Also, it must maintain stable haemodynamics during operation without need to increase blood transfusion. Lastly, an excellent anaesthetic must decrease recovery room stay by reducing post-operative pain, nausea, vomiting and requirement of additional analgesic.

PCNL can be done under General Anaesthesia or Spinal Anaesthesia. The most important advantages of Spinal anaesthesia are the decrease in intra-operative blood loss and consequently improving operating conditions, the decrease in peri-operative cardiac ischaemic incidents, post-operative hypoxic episodes, arterial and venous thrombosis and proper post-operative pain control.

Compared with patients receiving GA, patients receiving SA had higher haemoglobin levels on postoperative days 1 and 2 and a $20 \%$ lower total transfusion requirement. SA appears superior to GA for this procedure.[3]

The GA in PCNL procedure include its feasibility to control tidal volume and secure patient airway especially in prone position. The feasibility to control tidal volume minimises renal mobility secondary to respiration. ${ }^{[4]}$

Complications of general anaesthesia such as pulmonary (atelectasis), vascular and neurological disorders (Brachial 
nerve injury), especially during change of the position are more likely than the spinal anaesthesia.

The present cases were reported to highlight that in a select group of patients, PCNL under regional block is technically feasible and viable option. Regional block has the advantage of avoidance of general anaesthesia and anaphylaxis due to use of multiple drugs.[5]

\section{MATERIALS AND METHODS}

50 Patients, older than 15 years of age, who were undergoing Percutaneous Nephrolithotomy (PCNL) in American International Institute of Medical Sciences, Udaipur were selected for study. Intra-operative and post-operative haemodynamic study and anaesthetic complications, amount of blood loss and surgeon/patient satisfaction were studied.

All the patients received $10 \mathrm{mg}$ of metoclopramide and prophylactic antibiotics and were preloaded with $1000 \mathrm{~mL}$ of Ringer Lactate Solution.

Spinal anaesthesia was induced with Bupivacaine $15 \mathrm{mg}$, injected intrathecally at L4-5 or L3-4 space under all aseptic conditions and the head of table was tilted down for 5-6 minutes for fixing anaesthetic agents. The patient underwent cystoscopy and a ureteral catheter was placed under direct vision in lithotomy position.

Following the ureteral catheterisation, the patients were rotated to prone position for PCNL.

All patients underwent preoperative evaluation including detailed history taking, physical examination, preoperative urine analysis, urine culture, serum creatinine level, complete blood count (CBC) and liver function tests, electrocardiography (ECG) and plain chest x-rays. For the detection of stone characteristics, intravenous urography (IVU) and/or non-contrast computed tomography were carried out.

All patients received intravenous 3rd generation cephalosporin, 2 hrs. before surgery and for next 1 day thereafter. Intra-operative parameters included recording of pulse, blood pressure at basal level and every $15 \mathrm{~min}$. till the end of procedure. Hypotension was defined when systolic blood pressure was $<90 \mathrm{mmHg}$. Bradycardia was defined when pulse $<60$ beat/min. Any conversion from spinal to general anaesthesia was documented and the patient was excluded from the study. Operative time was calculated starting from onset of cystoscopic fixation of ureteric catheter till end of PCNL.

VAS score was recorded by attending nurse at $15 \mathrm{~min}$., 30 min., 1 hr., 2 hrs., 4 hrs., 6 hrs., 12 hrs., 18 hrs. and 24 hrs. postoperatively. Adverse effects including nausea, vomiting, shivering or pruritus were recorded up to $24 \mathrm{hrs}$. postoperatively. At the end of the study period, Satisfaction Visual Analogue Scale system was used to evaluate patients and surgeon satisfaction in a similar manner to that used to measure pain. The overall patients and surgeon satisfactions were assessed using a 10-point visual analogue scale (VAS) with 0 representing extremely unsatisfied and 10 representing extremely satisfied.
On first postoperative day, presence of any complications, and postoperative pain were checked. On the 2nd postoperative day, the nephrostomy tube was clamped for 3 hours. If there was no fever, urinary leak, of flank pain, nephrostomy tube would be removed and patient would be discharged. For 1 week if patient had any problem including headache, backache, lower limb pain, they were referred to the emergency room and standard treatment was started. Two weeks after the operation, all the patients underwent an ultrasonography for evaluation of the efficacy of operation and detection of residual calculi.

\section{RESULTS}

- The mean age of patients was $40 \pm 15$ years, the mean calculus size was $50.5 \pm 9.5 \mathrm{~mm}$.

- The mean operative time was $90 \pm 30$ minutes. Return of sensory and motor activity took $140 \pm 60$ minutes.

- 10 patients (20\%) developed Bradycardia which was treated with Atropine $0.5 \mathrm{mg}$ IV slowly.

- 5 patients (10\%) developed hypotension 3 to 10 minutes after the regional anaesthesia that was controlled by injecting $10 \mathrm{mg}$ ephedrine intravenously.

- According to VAS, 2 patients had (4\%) moderate-tosevere pain and 7 patients had mild (14\%) pain during the operation, which was controlled by $50 \mu \mathrm{g}$ Fentanyl and 25 mg of Ketamine.

- The mean haemoglobin decrease during 24 postoperative hours was $2 \pm 4 \mathrm{~g} / \mathrm{dL}$. 3 patients required transfusion of 1 unit of packed cell.

- The incidence of post-operative Nausea/Vomiting was observed in 5 patients.

- Surgeon's and Patient's satisfaction level was good after regional anaesthesia.

- 4 patients complained moderate post-subarachnoid puncture headache and dizziness and also mild low back pain, 3 to 7 days after operation, all of which improved by bed rest and conventional analgesics such as Acetaminophen and non-steroidal anti-inflammatory drugs.

- Post-operative shivering was observed in 8 patients.

\begin{tabular}{|c|c|c|}
\hline Sl. No. & Complication & No. of patients \\
\hline 1 & Bradycardia & $10(20 \%)$ \\
\hline 2 & Hypotension & $5(10 \%)$ \\
\hline 3 & Severe Peri-operative pain & $2(4 \%)$ \\
\hline 4 & Mild Peri-operative pain & $7(14 \%)$ \\
\hline 5 & $\begin{array}{c}\text { Blood loss requiring } \\
\text { transfusion }\end{array}$ & $3(6 \%)$ \\
\hline 6 & Nausea/Vomiting & $5(10 \%)$ \\
\hline 7 & Headache & $4(8 \%)$ \\
\hline 8 & Post-operative shivering & $8(16 \%)$ \\
\hline \multicolumn{3}{|c|}{ Table I. Complication of PCNL with } \\
\multicolumn{3}{|c|}{ Spinal Anaesthesia (n=50) } \\
\hline
\end{tabular}




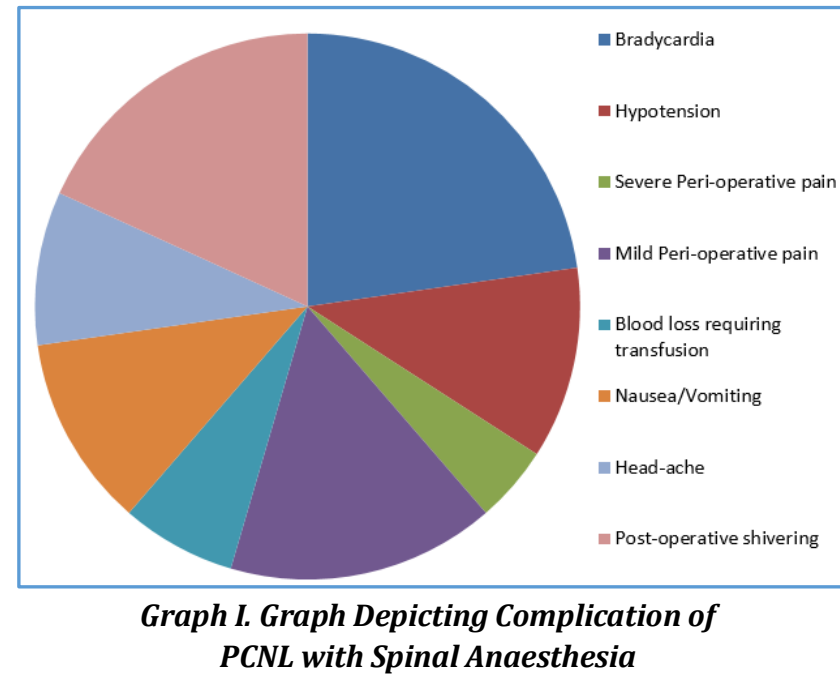

Major intra-operative or postoperative complications such as visceral, vascular, and neurologic injury or unusual bleeding did not occur in any of the patients.

\section{DISCUSSION}

PCNL is used for the fragmentation and removal of large and multiple calculi.

A good anaesthetic technique should have both rapid onset and reversal of effects. It should provide desirable intraoperative haemodynamic conditions, and if possible, contribute to a reduced need for blood transfusion. Moreover, it should permit the earliest possible discharge from the PACU and minimise the common postoperative problems such as pain, analgesics consumption, nausea, and vomiting.

Acute anaemia due to blood loss or dilution is a potent complication of PCNL that needs blood transfusion. Stoller and co-workers ${ }^{[6]}$ showed that the incidence of blood transfusion in single puncture PCNL reached 14, with an average decrease of $2.8 \mathrm{~g} / \mathrm{dL}$ in $\mathrm{Hb}$.

Several studies have shown that spinal anaesthesia results in less intra-operative bleeding compared with general anaesthesia. Solonia and colleagues[7] evaluated the impact of general anaesthesia versus spinal anaesthesia on intraoperative and post-operative outcome in patients undergoing radical prostatectomy. Moreover, spinal anaesthesia resulted in less intra-operative blood loss, less post-operative pain, and a faster post-operative recovery than general anaesthesia. Also, despite a small amount of mild and transient side effects, spinal anaesthesia was associated with significantly reduced blood loss, allowing a good haemodynamic and respiratory safety profile both intra and post-operatively.

In study by Maurer and co-workers, ${ }^{[3]}$ blood loss, operative time, and complications were compared in patient undergoing unilateral total hip replacement with either spinal or general anaesthesia. Compared with general anaesthesia, spinal anaesthesia resulted in a mean reduction of $12 \%$ in the operative time, $25 \%$ in intra-operative blood loss, $38 \%$ and $50 \%$ in intra-operative blood transfusion requirements.

There are a lot of debates whether Regional Anaesthesia is better than General Anaesthesia in PCNL or vice-versa.

Kuzgunbay et al found no difference between general anaesthesia and spinal epidural anaesthesia regarding operative time, postoperative haemoglobin level, hospital stay, success rate and postoperative complications. ${ }^{[8]}$
SA is usually associated with hypotension resulting from sympathetic block especially during changing into prone position. $[9,10,11]$

Several studies have also shown that spinal anaesthesia results in less intra-operative bleeding compared with general anaesthesia.[12,13] The disadvantages of general anaesthesia compared to regional spinal anaesthesia are increased incidence of anaphylaxis due to multiple medication usage and more pulmonary, vascular, neurologic complications and problems associated with the endotracheal tube during the change of position from lithotomy to prone. During supracostal puncture, patients with PCNL under regional anaesthesia can follow verbal commands and control respiration for prevention of pulmonary events. [14]

Fluid absorption was evident in all patients, although no patient had any clinical or biochemical evidence of intraoperative or postoperative electrolyte imbalance. This may be clinically significant in patients with compromised cardiorespiratory or renal status and in paediatric patients, leading to fluid overload. Fluid absorption may also be associated with both infective and non-infective pyrexia, necessitating adequate preoperative control of urinary infection. ${ }^{[15]}$

Creating a low-pressure system by using an Amplatz sheath, reducing the amount of irrigating fluid used, and staging the procedure significantly reduced the amount of fluid absorbed.[15]

The average blood loss for uncomplicated single puncture percutaneous nephrolithotomy was $2.8 \mathrm{~g} / \mathrm{dL}$ haemoglobin. Factors that potentially increase blood loss: Multiple punctures and/or renal pelvic perforation associated with a 2fold greater blood loss. Factors that did not affect blood loss: Calculus morphology, location, composition and length, number of fragments or stone-containing calices. Other factors such as puncture site, type of fascial dilation, hypertension, renal insufficiency, infection, previous open renal surgery or previous extracorporeal shock wave lithotripsy, also did not affect total estimated blood loss.

Half of the expected blood loss occurred in patients with pre-existing nephrostomy tract.

\section{CONCLUSION}

Spinal anaesthesia could be a very good method of anaesthesia for percutaneous nephrolithotomy with trivial pain, less blood loss and without major complications; and also surgeon's and patient's satisfaction is more. Thus, it may be a good alternative of General Anaesthesia for performing PCNL in adult patients. Further studies may be carried out to statistically prove that Spinal Anaesthesia may be a better alternative to General Anaesthesia.
Abbreviations
L4-5: Space between Lumbar vertebrae 4 and 5.
VAS: Visual Analogue Scale.
PCNL: Percutaneous Nephrolithotomy.
SA: Spinal Anaesthesia.
GA: General Anaesthesia.
RA: Regional Anaesthesia. 


\section{REFERENCES}

1. Wong MY. Evolving technique of percutaneous nephrolithotomy in developing country: Singapore general hospital experience. J Endourol 1998;12(5):397401.

2. Rozentsveig V, Neulander EZ, Roussabrov E, et al. Anaesthetic considerations during percutaneous nephrolithotomy. J Clin Anesth 2007;19(5):351-5.

3. Maurer SG, Chen AL, Hiebert R, et al. Comparison of outcomes of using spinal versus general anaesthesia in total hip arthroplasty. AM J Orthop (Belle Mead NJ) 2007;36(7):E101-6.

4. El Moawad SH, El Hefnawy AS. Spinal vs. general anesthesia for percutaneous nephrolithotomy: a prospective randomized trial. Egyptian Journal of Anaesthesia 2015;31(1):71-5.

5. Singh I, Kumar A, Kumar P. Ambulatory PCNL (tubeless PCNL under regional anaesthesia) - a preliminary report of 10 cases. Int urolnephrol 2005;37(1):35-7.

6. Stoller ML, Wolf JS, St Lezin MA. Estimated blood loss and transfusion rates associated with percutaneous nephrolithotomy. J Urol 1994;152(6 Pt 1):1977-81.

7. Salonia A, Suardi N, Crescenti A, et al. General versus spinal anesthesia with different forms of sedation in patients undergoing radical retropubic prostatectomy: results of a prospective randomized study. Int J Urol 2006;13(9):1185-90.

8. Kuzgunbay B, Turunc T, Akin S, et al. Percutaneous nephrolithotomy under general versus combined spinalepidural anesthesia. J Endourol 2009;23(11):1835-8.
9. Mehrabi S, Shirazi KK. Results and complications of spinal anesthesia in percutaneous nephrolithotomy. Urol J 2010;7(1):22-5.

10. Sunana G, Rahul G, Nandita M, et al. Percutaneous nephrolithotomy under spinal anesthesia and the efficacy of adding adjuvant clonidine to intrathecal hyperbaric bupivacaine: a comparative study. Internet J Anesthesiol 2014;33(1):2.

11. Movasseghi G, Hassani V, Mohaghegh MR, et al. Comparison between spinal and general anesthesia in percutaneous nephrolithotomy. Anesth Pain Med 2014;4(1):e13871.

12. Shir Y, Raja SN, Frank SM, et al. Intraoperative blood loss during radical retropubic prostatectomy: epidural versus general anesthesia. Urology 1995;45(6):993-9.

13. Davis FM, McDermott E, Hickton C, et al. Influence of spinal and general anaesthesia on haemostasis during total hip arthroplasty. Br J Anaesth 1987;59(5):561-71.

14. Singh V, Sinha RJ, Sankhwar SN, et al. A prospective randomized study comparing percutaneous nephrolithotomy under combined spinal-epidural anesthesia with percutaneous nephrolithotomy under general anesthesia. Urol Int 2011;87(3):293-8.

15. Kukreja RA, Desai MR, Sabnis RB, et al. Fluid absorption during percutaneous nephrolithotomy: does it matter? J Endourol 2002;16(4):221-4. 